\title{
Modeling dark haloes in early-type galaxies: stellar kinematics at large radii
}

\author{
Anne-Marie Weijmans ${ }^{1,2}$ \\ ${ }^{1}$ Sterrewacht Leiden, Leiden University, Postbus 9513, 2300 RA Leiden, the Netherlands \\ email: weijmans@strw.leidenuniv.nl \\ ${ }^{2}$ European Southern Observatory, Karl-Schwarzschild-Str 2, 85748 Garching, Germany
}

We developed a new method to obtain absorption line spectra of early-type galaxies at large radii, using integral-field spectrography (IFS). By using the spectrograph as a 'photon-collector' and adding the signal of many individual spaxels together in one spectrum, we obtain sufficient signal-to-noise to measure both stellar kinematics and line strengths at large radii. These can be used to determine the properties of the dark matter halo, as well as the stellar halo population.

We applied this method to the early-type galaxies NGC 3379 and NGC 821, using the SAURON IFS (Weijmans et al. 2009). We sampled the major axis of these galaxies with the IFS. The resulting stellar kinematics agree with data from long-slit spectrography and planetary nebulae. We construct mass models using the triaxial Schwarzschild code developed by van den Bosch et al. (2008) and find that inclusion of a dark halo is necessary for both systems to explain the observed kinematics. At least $30-50 \%$ of the total matter within $4 R_{e}$ is dark.

We also observed the early-type galaxy NGC 2549 with the PPak IFS, but instead of sampling the major axis, we obtained a full mosaic out to almost $5 R_{e}$ (Weijmans 2009). We find that NGC 2549 rotates in a disc-like structure over the total observed field. For this galaxy we also confirm the presence of a dark halo, with a dark matter fraction of at least $63 \%$ within $5 R_{e}$.

\section{References}

van den Bosch, R. C. E., van de Ven, G., Verolme, E. K., Cappellari, M., \& de Zeeuw, P. T., 2008, MNRAS, 385, 647

Weijmans, A., 2009, PhD thesis, Leiden University

Weijmans, A., et al., 2009, MNRAS, in press (arXiv0908.0018) 